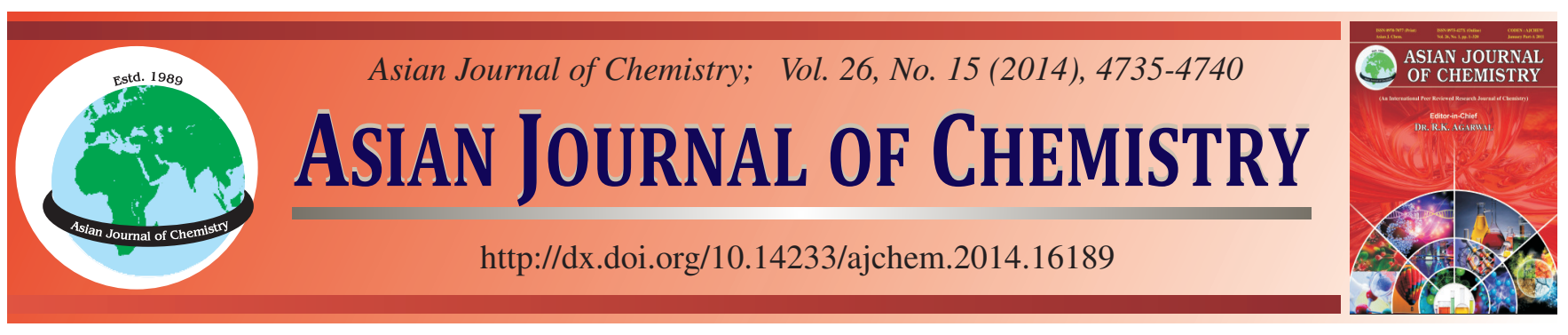

\title{
Preparation and Properties of Branched Poly(phosphoric ester) Oligomer and its Influences on Poly(L-lactide)
}

\author{
YuQING BAi, YingBin LiU and Mingtao Run ${ }^{*}$
}

College of Chemistry \& Environmental Science, Hebei University, Baoding, P.R. China

*Corresponding author: Fax: +86 312 5079386; Tel: +86 312 5079359; E-mail: 1hbx@ @bu.edu.cn

\begin{abstract}
Branched poly(phosphoric ester) (BPPE) oligomer containing intumescent flame retardant were synthesized from spirocyclic pentaerythritol diphosphonate dichloride (SPDPC) and triethanolamine (TEA) and it was blended with poly(L-lactide) (PLLA) to investigate its influences on the thermal and rheological properties of poly(L-Lactide). The results suggest that branched poly(phosphoric ester) oligomer is an intumescent flame retardant whose residual char reaches $47 \%$ at $600{ }^{\circ} \mathrm{C}$ and it can partially crystallize into monoclinic crystals. When it was blended with poly(L-lactide), it functions as an intumescent flame retardant, not only widening the degradation temperature range at high temperature but also increasing the residual carbon ratio of poly(L-lactide). Branched poly(phosphoric ester) also serves as a plasticizer for poly(L-lactide) by decreasing the melt viscosity.
\end{abstract}

Keywords: Branched oligomer, Intumescent flame retardant, Thermal stability, Melting, Rheology.

\section{INTRODUCTION}

Nowadays, the intumescent flame retardant has attracted a widespread attention due to its advantages, such as low toxicity, low smoke and non-corrosive $\mathrm{e}^{1-4}$. Compared with small molecular multi-component flame retardant, single-component intumescent flame retardant itself plays a role of carbon source, acid source and gas source. With the less content, better thermal stability and better resistance to hygroscopicity, it has been paid more and more attention. Haipern et al..$^{5}$ prepared pentaerythritol diphosphonate of melamine (PDM) from spirocyclic pentaerythritol diphosphonate dichloride (SPDPC) and melamine. Compared with the other flame retardant, PDM has better thermal stability and chemical stability due to its spirocyclic structure.

Previous results showed that linear polymers containing SPDPC could improve the stability of the materials effectively. Ma et $a l .^{6}$ used SPDPC and 4,4'-diaminodiphenyl methane (DDM) to prepare poly(4,4-diaminodiphenyl methane spirocyclic pentaerythritol bisphosphonate) (PDSPB), which was used to modify acrylonitrile-butadiene-styrene (ABS). The results showed that the addition of PDSPB enhanced the thermal stability and flame retardancy of ABS significantly. Wang et al. ${ }^{7}$ synthesized poly (DOPO substituted dihydroxyl phenyl pentaerythritol diphosphonate) (PFR) and blended it with epoxy resin (EP). The results showed the incorporation of PFR into EP can improve the thermal stability and flame retardancy dramatically. Chen et al. ${ }^{8}$ prepared poly(2-hydroxy propylene spirocyclic pentaerythritol bisphosphonate) and blended it with poly(ethylene terephthalate) fibrics. The results showed that the fibrics had improved flame retardancy and dripping resistance.

Branched polymer (BP) is a polymer of a special molecular structure ${ }^{9-12}$. Compared to linear polymer, branched polymer has high solubility and compatibility with other materials, as well as a lower viscosity ${ }^{13}$. Branched polymer could be act as a surface modification agent and a toughening agent of the materials and coatings ${ }^{14-16}$.

If the spirocyclic structure can be combined with branched structure to prepare a kind of branched polymer, it is believed that the branched polymer will has intumescent flame retardancy, low melt viscosity, larger molecular weight, better thermal stability and better compatibility with other polymers. Poly(L-lactide) has been intensively studied and widely used for biomedical materials because of its high biocompatibility and good biodegradability in the earth's environment ${ }^{17}$. However, poly(L-lactide) fall short of the required properties for potential applications, such as higher processing viscosity ${ }^{18}$, lower flame retardancy, lower toughness ${ }^{19}$, etc. In this work, in order to improve the processing properties and flame retardancy of poly(L-lactide), a kind of branched poly(phosphoric ester) oligomer containing the spirocyclic structure was synthesized and blended with poly(L-lactide) and the effects of BPPE on the thermal and rheological properties of poly(L- 
lactide) were investigated and characterized by various measurements. The results suggest that BPPE can serve both as a flame intumescent retardant and a plasticizer for poly $(\mathrm{L}-$ lactide).

\section{EXPERIMENTAL}

Phosphoryl chloride, CR, Kelong Chemical Reagent Co. (Chengdu, China); pentaerythritol, AR, Hongxie Chemical Reagent Co. (Beijing, China); Triethanolamine, AR, Xintong Fine Chemical Reagent Co. Ltd (Tianjing, China). Poly(Llactide), Lacty9000, $\mathrm{T}_{\mathrm{m}}=174.6^{\circ} \mathrm{C}$, Shimadzu Co. Japan.

General procedure for the synthesis of SPDPC: Spirocyclic pentaerythritol diphosphonate dichloride was synthesized according to the reported methods ${ }^{20,21}$. Some characterizations of SPDPC were carried out by fourier transform infrared (FTIR) spectroscopy and ${ }^{1} \mathrm{H}$ nuclear magnetic resonance $\left({ }^{1} \mathrm{H}\right.$ NMR) (appendix). FTIR $\left(\mathrm{KBr}, \mathrm{v}_{\max }, \mathrm{cm}^{-1}\right)$ : 1307 $(\mathrm{P}=\mathrm{O}), 1031$ (P-O-C), 852 (P-O), 582, 551 (P-Cl); ${ }^{1} \mathrm{H}$ NMR (600 MHz, DMSO- $\left.d_{6}, \mathrm{ppm}\right): \delta 4.23-4.21\left(\mathrm{~d}, 8 \mathrm{H}, \mathrm{OCH}_{2}\right)$.

General procedure for the synthesis of branched poly (phosphoric ester): Branched poly(phosphoric ester) (BPPE) oligomer was synthesized by the method shown in Scheme-I. 8.91g (0.03 mol) SPDPC, 8.94g (0.06 mol) triethanolamine and $200 \mathrm{~mL}$ dimethylbenzene were added in a $500 \mathrm{~mL}$ threeneck round flask containing a magnetic stirrer and equipped with a nitrogen inlet, a thermometer, a reflux condenser and a heating oil bath. The flask was heated up to $50{ }^{\circ} \mathrm{C}$, then stirred for $18 \mathrm{~h}$ under the protection of nitrogen atmosphere. At last, the solution was cooled to room temperature and filtered the precipitate. The yellow powder was collected and dried to constant weight at $50{ }^{\circ} \mathrm{C}$ in a vacuum oven.

FTIR (KBr, $\left.\mathbf{v}_{\max }, \mathbf{c m}^{-1}\right)$ : 3400-3300 (-OH), $1317(\mathrm{P}=\mathrm{O})$, 1037 (P-O-C), 852 (P-O); ${ }^{1} \mathrm{H}$ NMR (600 MHz, DMSO-d $d_{6}$ ppm): $\delta 4.63$ (s, $1 \mathrm{H}, \mathrm{OH}), 4.21-4.25\left(\mathrm{~d}, 8 \mathrm{H}, \mathrm{OCH}_{2}\right), 3.72-3.85$ (t, 2H, $\mathrm{CH}_{2}$ ), 3.64-3.68 (t, 2H, $\left.\mathrm{CH}_{2}\right), 3.28-3.36\left(\mathrm{t}, 2 \mathrm{H}, \mathrm{CH}_{2}\right)$ (appendix). Elemental analysis: experimental values, C, 31.37; H, 5.75; N, $3.97 \%$; theoretical values, C, 29.59; H, 4.48; N, $3.14 \%$. Mass spectrometry $\left(\mathrm{DOOCF}_{3}\right)$ : molecular weight is in the range of 2672 and 5894.

FTIR, ${ }^{1} \mathrm{H}$ NMR, elemental analysis and the mass spectrometry results suggest that the branched poly(phosphoric ester) oligomer containing SPDPC had been synthesized, as shown<smiles>O=P1(Cl)OCC2(CO1)COP(=O)(Cl)OC2</smiles>

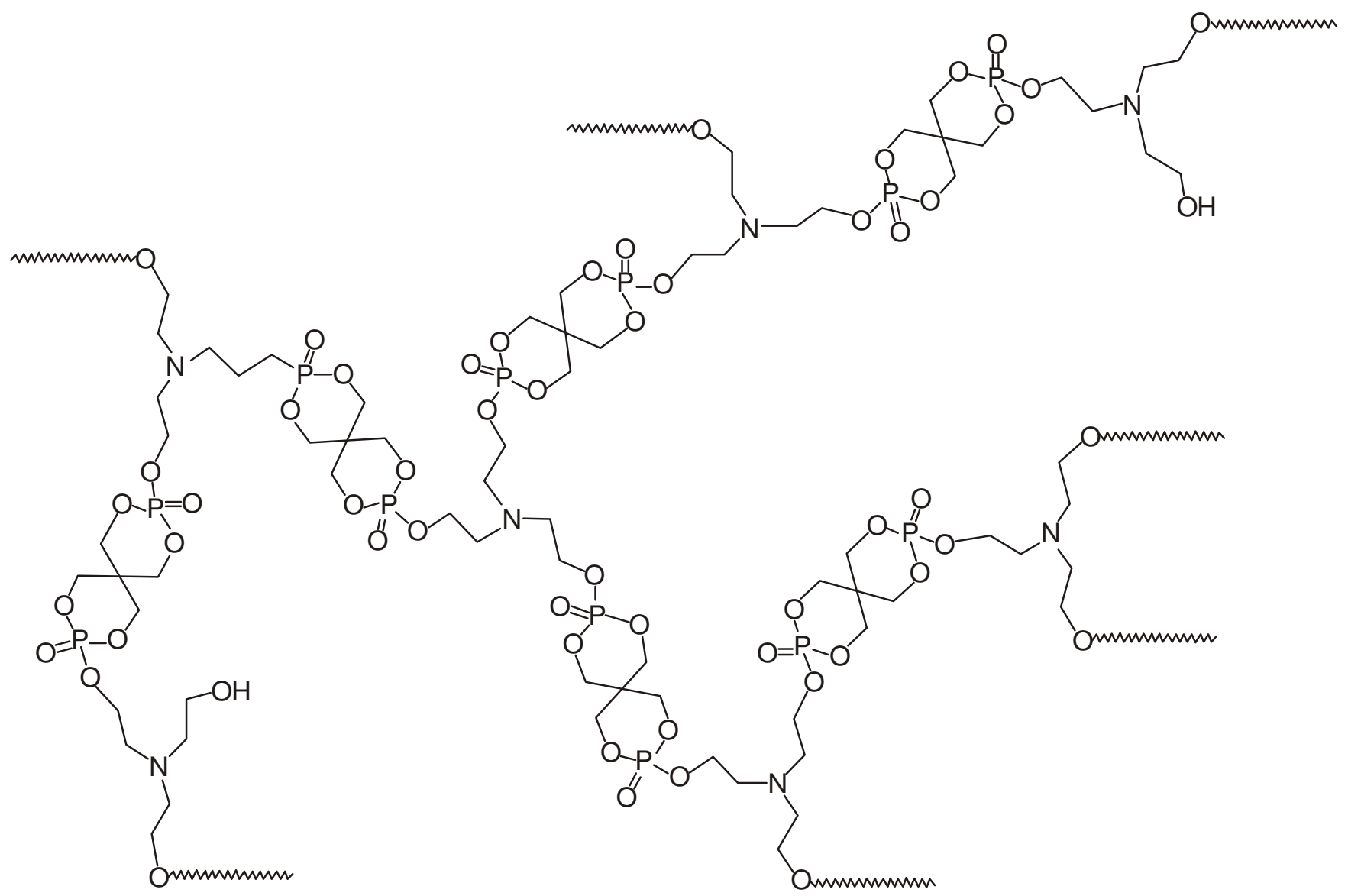

Scheme-I: Synthesis of branched poly(phosphoric ester) via the polymerization of SPDPC and triethanolamine 
in Scheme-I. However, the high molecular weight polymer had not been obtained. It may be because that the dimethylbenzene becomes bad solvent for the product BPPE with increasing molecular weight and BPPE precipitates from the solvent and its further solution propagation reaction stops. Although some branch chains form in the oligomer, a few -OH end-groups don't react and only form a few linear short chains. Although only the oligomer is obtained, it is deduced that BPPE can have some advantages of both the intumescent flame retardancy and the branched polymers, such as the relative low melt-viscosity, high polarity, etc.

Preparation of poly(L-lactide)/BPPE blend: The blend of poly(L-lactide)/BPPE (w/w = 90/10) was prepared by melt-blended method using a SJ-15 type, co-rotating twintaperscrew micro-extruder with one heating section, made by Wuhan Ruiming Machinery Co. (China), operating at a screw speed of $70 \mathrm{rpm}$ and a temperature at $190{ }^{\circ} \mathrm{C}$. The resultant blend ribbons were cooled in cold water, cut up and re-dried before being used in measurements.

FTIR spectra were measured on a Varian 640 FTIR spectrometer (Varian 640, Varian Co., US), using the $\mathrm{KBr}$ pellet technique. ${ }^{1} \mathrm{H}$ NMR spectra were performed at on a Bruker Avance $600 \mathrm{NMR}$ spectrometer $(600 \mathrm{MHz})$ operating in the Fourier transform mode using DMSO- $d_{6}$ as the solvent and tetramethylsilane as an internal standard. The quantitative analyses of $\mathrm{C}, \mathrm{H}, \mathrm{N}$ and $\mathrm{O}$ were carried out on an exeter analytical CE-440 elemental analyzer. The molecular weight was measured by high resolution fourier transform mass spectrometry (FTMS, Ultra 7.0,) using $\mathrm{DOOCF}_{3}$ as solvent.

The thermal stability of the BPPE and poly(L-lactide)/ BPPE blend was studied by the thermogravimetric analyzer (TGA) (Pyris 6, Perkin-Elmer Co., USA) in the following manner: the sample was heated from 30 to $800{ }^{\circ} \mathrm{C}$ at a rate of $20{ }^{\circ} \mathrm{C} / \mathrm{min}$ under oxygen atmosphere and the degradation process was recorded.

The melting and glass transition behaviours of branched poly(phosphoric ester) were studied by DSC (Diamond, Perkin-Elmer Co. US) in the following manner: the sample was heated from -60 to $180{ }^{\circ} \mathrm{C}$ at a rate of $100^{\circ} \mathrm{C} / \mathrm{min}$ under a nitrogen atmosphere and held for $3 \mathrm{~min}$ to remove the thermal history; then the melt was cooled to $-70{ }^{\circ} \mathrm{C}$ at a rate of $80^{\circ} \mathrm{C} / \mathrm{min}$ and then heated from $-70{ }^{\circ} \mathrm{C}$ to $180^{\circ} \mathrm{C}$ at a rate of $10^{\circ} \mathrm{C} / \mathrm{min}$. The final heating process was recorded.

Wide-angle X-ray diffraction (WAXD) testing was carried out using X-ray diffractometer (D8 Advance, Bruker, Germany). Nickel-filtered $\mathrm{CuK}_{\alpha}(\lambda=0.15418 \mathrm{~nm})$ radiation generated at $40 \mathrm{kV}$ and $40 \mathrm{~mA}$ was used. The diffraction curves were recorded as $2 \theta$ scans in the range of $10-60^{\circ}$ at a scanning speed of $10 \%$ min using a step size of $0.02^{\circ}$.

The samples were first melted between two glass slides on a hot stage at $148{ }^{\circ} \mathrm{C}$ for $5 \mathrm{~min}$, pressed into a film, then cooled to the temperature at $1{ }^{\circ} \mathrm{C} / \mathrm{min}$ until the nuclei appeared, held at the temperature for $4 \mathrm{~h}$. Using polarized optical microscopy (BX-51, Olympus, Japan) to observe the crystal morphology and take photos by the digital camera.

Dynamic mechanical properties of branched poly(phosphoric ester) were studied by a dynamic mechanical analyzer (DMA, 8000, Perkin-Elmer Co., USA). DMA scans were carried out from -80 to $140{ }^{\circ} \mathrm{C}$ using a single-cantilever vibration mode at a constant heating rate of $2{ }^{\circ} \mathrm{C} / \mathrm{min}$ and a frequency of $1 \mathrm{~Hz}$.

The dried poly(L-lactide)/BPPE blend ribbon was burned in air and then crushed out by blow and the burnt surface was coated with a thin layer of gold and then investigated using a KYKY-2800B type scanning electron microscope (KYKY Technology Development Ltd. Co., China) at a voltage of $25 \mathrm{kV}$.

The dynamic rheology measurements of the poly $(\mathrm{L}-$ lactide) and poly(L-lactide)/BPPE blend were determined at $180{ }^{\circ} \mathrm{C}$ by an AR2000ex type rotational rheometer (WatersTA Co., USA) with two parallel plates with diameter of $25 \mathrm{~mm}$ and $1.0 \mathrm{~mm}$ gap width. The frequencies were changed from 0.628 to $628 \mathrm{rad} / \mathrm{s}$.

\section{RESULTS AND DISCUSSION}

Fig. 1 showed TG and DTG curves of BPPE. TG curve shows that BPPE starts degradation at $229.8{ }^{\circ} \mathrm{C}\left(\mathrm{T}_{2 \%}\right)$ in nitrogen environment. Three main decomposition stages were observed in DTG curve in Fig. 1. The first stage occurs in the temperature range of $120-287^{\circ} \mathrm{C}$ and its weight loss is $11 \%$. The second and third stages occurs in the temperature range of $287-432{ }^{\circ} \mathrm{C}$ and $432-800{ }^{\circ} \mathrm{C}$ with the weight loss of 33 and $16 \%$, respectively. Finally, the residue of BPPE is $47 \%$ at $600{ }^{\circ} \mathrm{C}$. It suggests that the BPPE is an efficient charforming agent. DTG curve also indicates the BPPE has a minor (at about $277^{\circ} \mathrm{C}$ ) and a major (at about $349^{\circ} \mathrm{C}$ ) weight-loss stage which can be assigned to the scission of the phosphate ester bonds (minor stage) and intumescent char formation (major stage). The residual char can prevent further degradation of materials ${ }^{21}$.

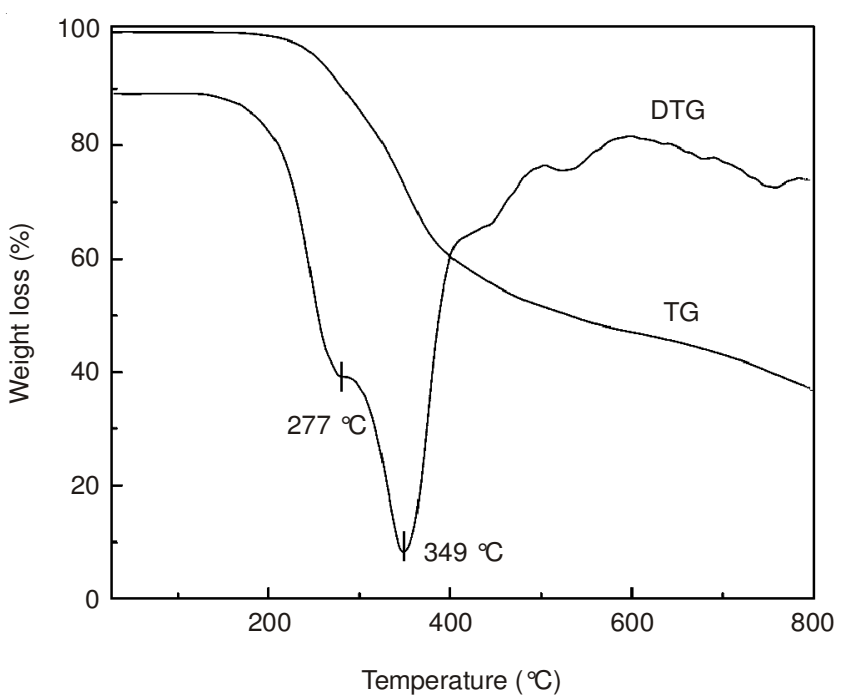

Fig.1. TG and DTG curves of BPPE

Melting behaviour: Fig. 2 shows the DSC heating curve of the quenched BPPE at a rate of $10^{\circ} \mathrm{C} / \mathrm{min}$. The glass transition temperature $\left(\mathrm{T}_{\mathrm{g}}\right)$ and melting point $\left(\mathrm{T}_{\mathrm{m}}\right)$ can be observed at -12.7 and $146{ }^{\circ} \mathrm{C}$ in the heating process, respectively. Moreover, a recrystallization behavior is also observed in the curve with the peak temperature of $89.7^{\circ} \mathrm{C}$. When the BPPE was melton and quenched by ice-water, some microcrystallites 
formed; in the heating process, these microcrystallites melt and then recrystallize into bigger crystals in the heating process and then these bigger crystals melt at melting point. These results suggest that the BPPE oligomer can crystallize into crystal.

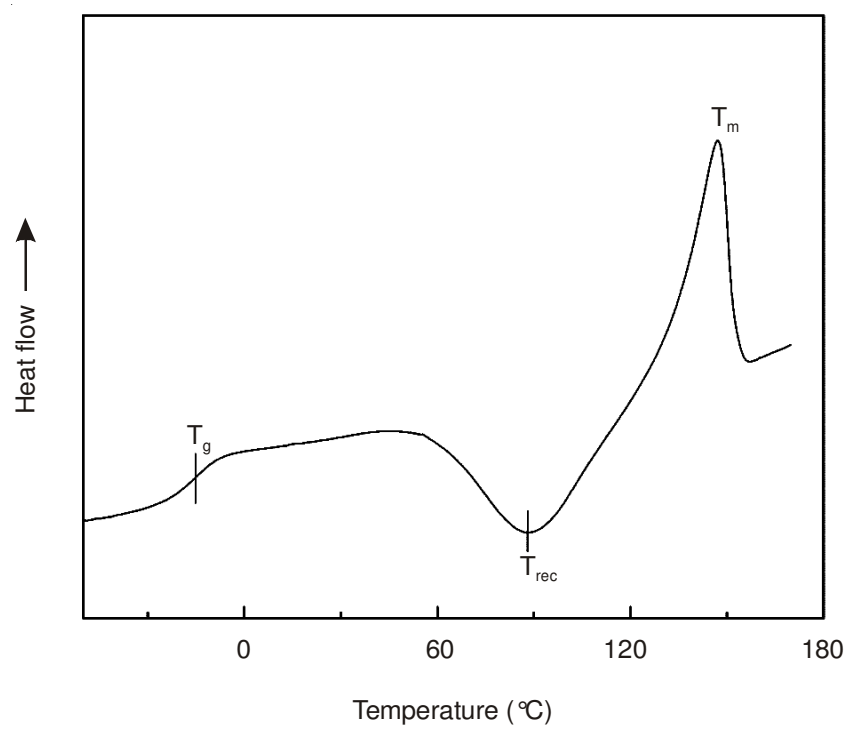

Fig. 2. DSC heating curve of BPPE

Dynamic mechanical analysis: Fig. 3 shows the curves of storage modulus (E') and $\tan \delta v$. T of BPPE. The storage modulus of the BPPE is $12 \times 10^{6} \mathrm{MPa}$ at $-60{ }^{\circ} \mathrm{C}$. The storage modulus of the BPPE declines dramatically between $-30{ }^{\circ} \mathrm{C}$ and $90{ }^{\circ} \mathrm{C}$. The $\tan \delta$ reach maximum at $-5^{\circ} \mathrm{C}$, corresponding to the glass transition temperature of BPPE. The shoulder peak in the temperature range of 20 to $50{ }^{\circ} \mathrm{C}\left(\mathrm{T}_{\mathrm{re}}\right)$ corresponds to the recrystallization behaviour of the $\mathrm{BPPE}$, which consists with the result of DSC.

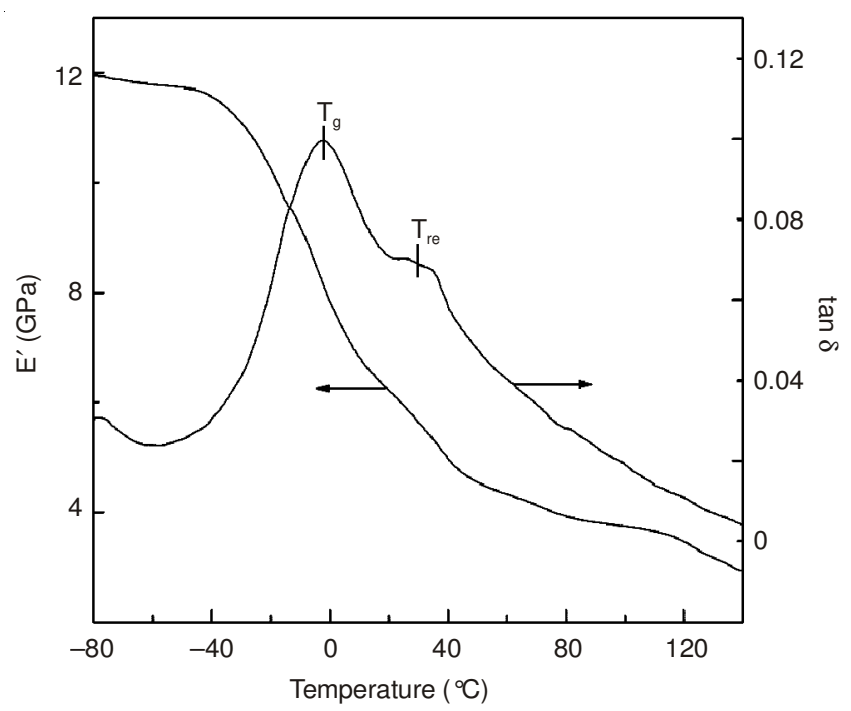

Fig. 3. Curves of storage modulus and $\tan \delta v s$. temperature of BPPE

Crystal morphology: When the melton BPPE oligomer was cooled from $148{ }^{\circ} \mathrm{C}$ and annealed at $106{ }^{\circ} \mathrm{C}$, BPPE can form crystals, as shown in Fig. 4 and the crystal morphology represents regular shape. Fig. 5 shows X-ray diffraction curve of BPPE oligomer. The result shows that the crystal is monoclinic.

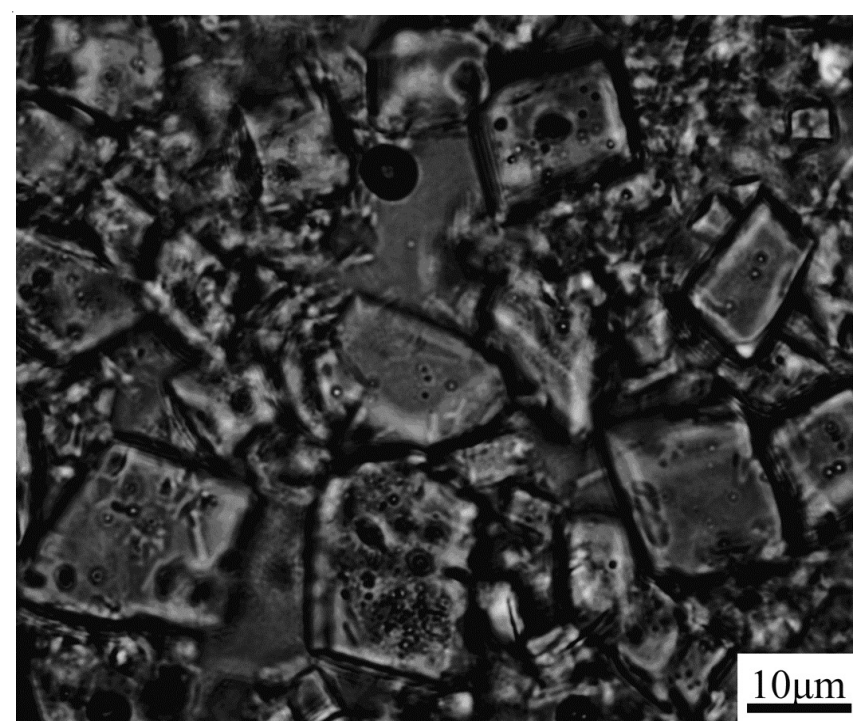

Fig. 4. POM micrographs of crystallized samples

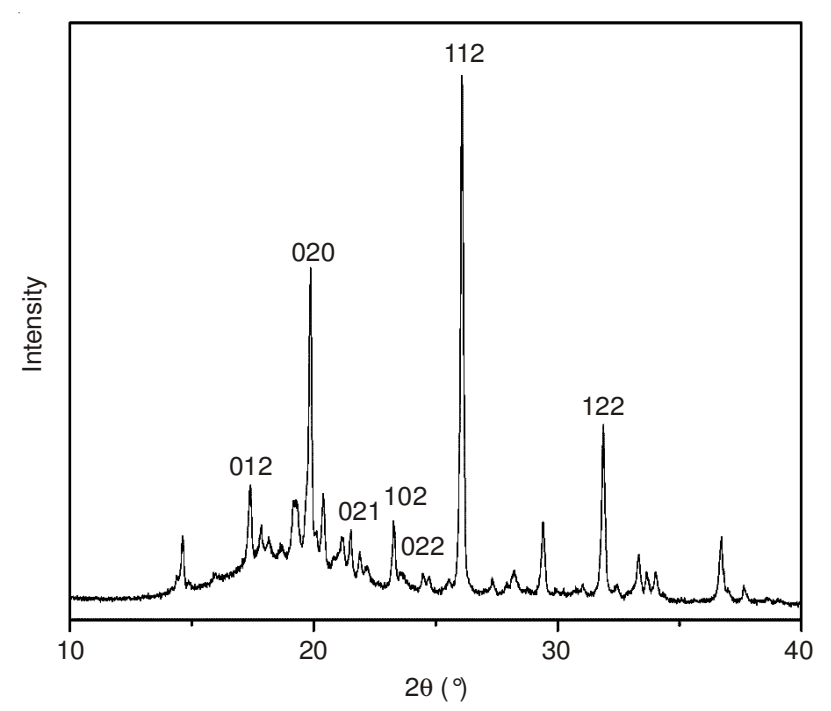

Fig. 5. X-ray diffraction curves of BPPE

Thermal degradation of poly(L-lactide)/BPPE blend: Thermal degradation behaviours of poly(L-lactide) and poly(Llactide)/BPPE blend were shown in Fig. 6. Both the curves show one predominant degradation stage. In the TG curves, poly(L-lactide) shows higher thermal stability with a $\mathrm{T}_{2 \%}$ at $337^{\circ} \mathrm{C}$, while the blend gives a lower $\mathrm{T}_{2} \%$ at $306^{\circ} \mathrm{C}$. The reason may because that the P-O and P-C bonds of BPPE have low bond energy, which degrades at low temperatures. Both the curves have a small second degradation stages, which is caused by the further degradation of the residual carbons. However, in the DTG curves, although poly(L-lactide) and poly(Llactide)/BPPE have the same $\mathrm{T}_{\max }$ at $384{ }^{\circ} \mathrm{C}$ in the first degradation stages, poly(L-lactide)/BPPE blend has a higher second degradation peak at $498{ }^{\circ} \mathrm{C}$ than that of poly(L-lactide) at $467{ }^{\circ} \mathrm{C}$. Moreover, the residual carbon weight percent of 
poly(L-lactide)/BPPE is $7.1 \%$ while that of poly(L-lactide) is $0 \%$ at $600{ }^{\circ} \mathrm{C}$. The increased temperature range of the second degradation stage and the increased residual carbon suggest that BPPE improves the thermal stability of poly(L-lactide) at high temperatures and BPPE induces poly(L-lactide) forming more residual carbons and decreases the amount of the combustible gas and functions as a flame retardant by forming agglomerate phase ${ }^{22}$.

The residual carbon surfaces morphology of the burnt poly(L-lactide) and poly(L-lactide)/BPPE blend were shown in Fig. 7. In Fig.7a, the burnt surface of poly(L-lactide) is relative smooth; while for poly(L-lactide)/BPPE in Fig. 7b, it has many cavities and heaves on the surface, which is because that the volatile substances formed as poly(L-lactide) and BPPE decomposing breaks through the burning surface. These intumescent carbon foam surface can not only prevent the further degradation of poly(L-lactide) but also retard the oxygen and heat transfer to the burning surface, as a result, the flame spread is retarded. The foams on surface also suggest that BPPE is a kind of intumescent flame retardant.

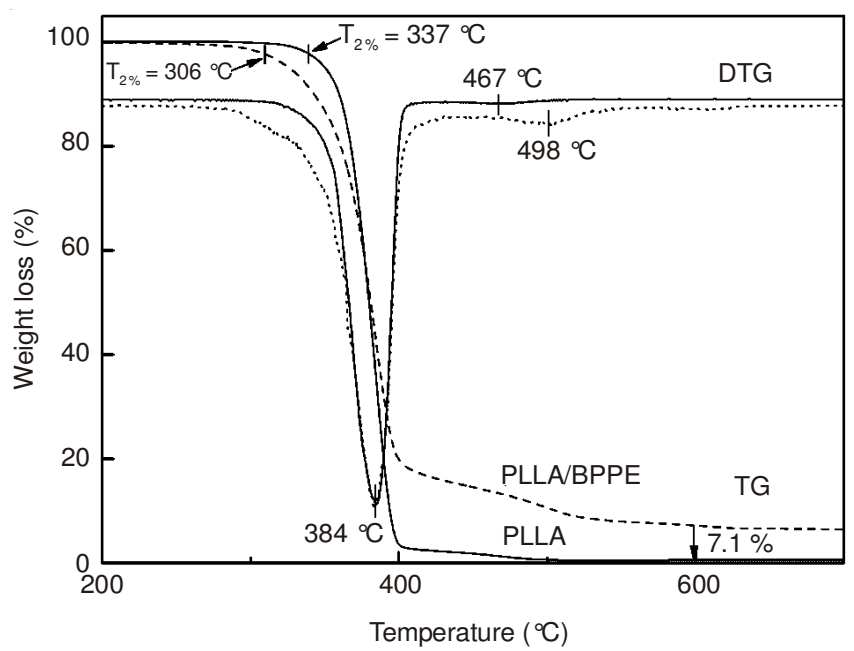

Fig. 6. TG and DTG curves of poly(L-lactide) and poly(L-lactide)/BPPE

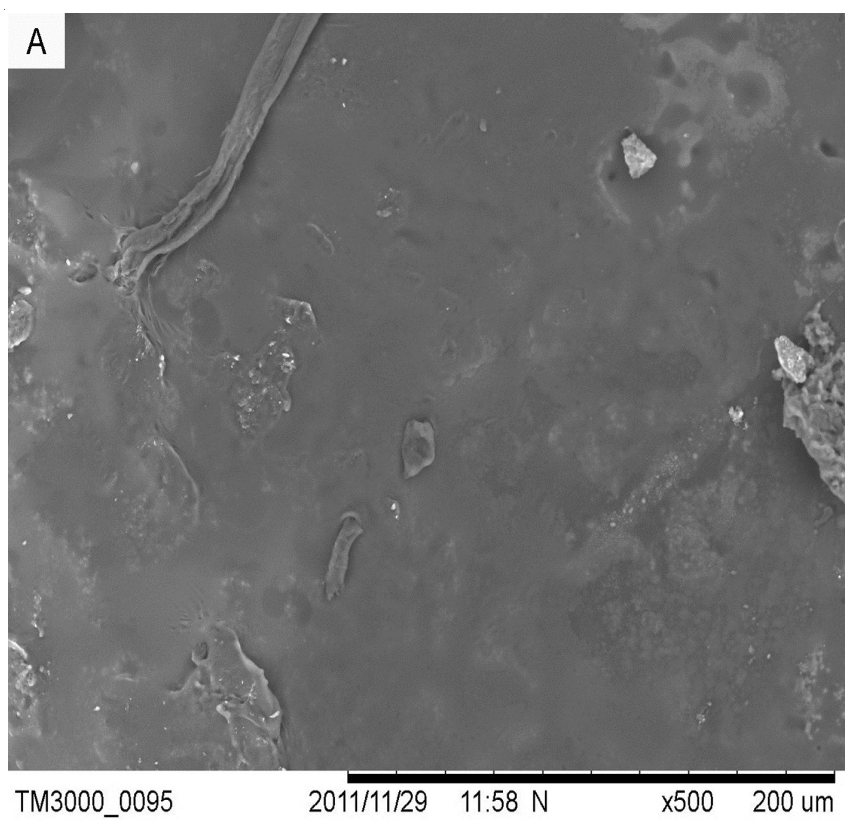

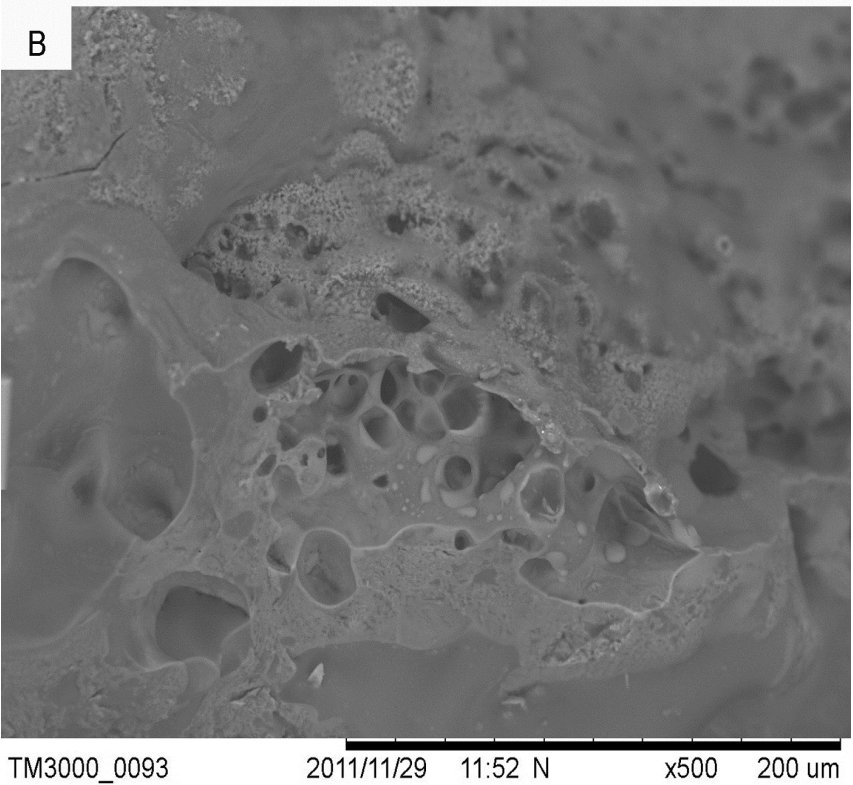

Fig. 7. SEM morphology of residual carbon of poly(L-lactide) and poly(Llactide)/BPPE

Rheology behaviour of poly(L-lactide) and poly(Llactide)/BPPE blend: In order to characterize the influence of BPPE on the melt viscosity of poly(L-lactide), the rotational rheology of the samples was measured. Fig. 8 exhibits the complex viscosities $(\eta *)$ of poly(L-lactide) and poly(Llactide)/BPPE blend $v s$. the frequency. Fig. 8 showed that a significant drop in the blend viscosity occurred apparently on addition of BPPE. This result suggests that the melton BPPE oligomer acts as a plasticizer for poly(L-lactide) due to BPPE's low melt viscosity.

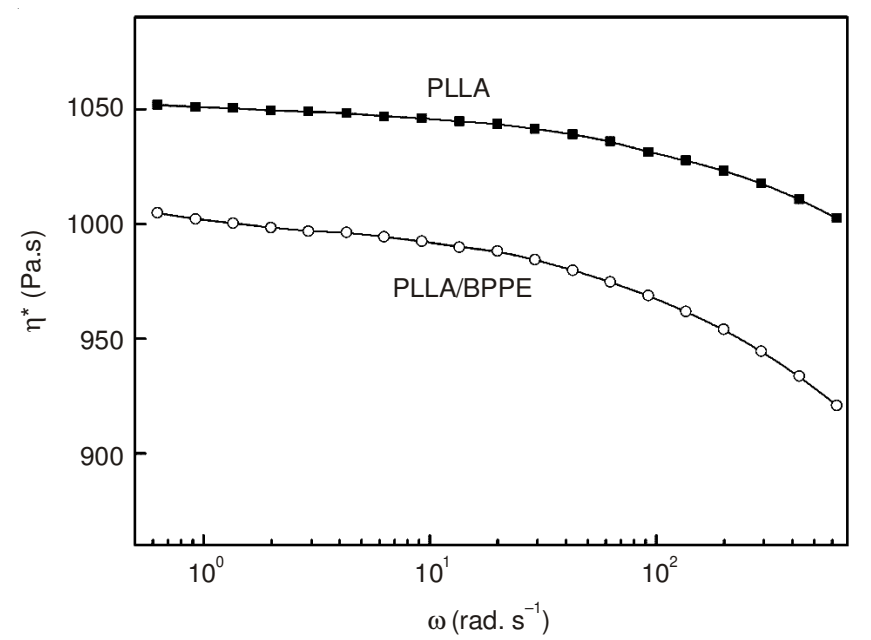

Fig. 8. Effect of BPPE on the complex viscosity of poly(L-lactide) at $180{ }^{\circ} \mathrm{C}$

\section{Conclusion}

A poly(phosphoric ester) (BPPE) oligomer containing intumescent flame retardant were synthesized from spirocyclic pentaerythritol diphosphonate dichloride (SPDPC) and triethanolamine. Branched poly(phosphoric ester) is an intumescent flame retardant whose residual char reaches $47 \%$ at $600{ }^{\circ} \mathrm{C}$. When it is blended with poly(L-lactide), it functions not only as an intumescent flame retardant by increasing the 
degradation temperature range and the residual carbon ratio of poly(L-lactide) but also serves as a plasticizer for poly(Llactide) by decreasing the melt viscosity.

\section{ACKNOWLEDGEMENTS}

The authors are grateful for support by the Natural Science Foundation of Hebei Province (Contract grant number: B2010000219).

\section{REFERENCES}

1. M. Gao and S.S. Yang, J. Appl. Polym. Sci., 115, 2346 (2010).

2. A.K. Sen, B. Mukherjee, A.S. Bhattacharya, L.K. Sanghi, P.P. De and A.K. Bhowmick, J. Appl. Polym. Sci., 43, 1673 (1991).

3. Z.L. Ma, M. Zhao, H.F. Hu, H.T. Ding and J. Zhang, J. Appl. Polym. Sci., 83, 3128 (2002).

4. Y. Chen, Y. Liu, Q. Wang, H. Yin, N. Aelmans and R. Kierkels, Polym. Degrad. Stabil., 81, 215 (2003).

5. Y. Halpern, D.M. Mott and R.H. Niswander, Ind. Eng. Chem. Prod. Res. Dev., 23, 233 (1984).

6. H.Y. Ma, L.F. Tong, Z.B. Xu, Z.P. Fang, Y.M. Jin and F.Z. Lu, Polym. Degrad. Stabil., 92, 720 (2007).
7. X. Wang, Y. Hu, L. Song, W.Y. Xing, H.D. Lu, P. Lv and G.X. Jie, Polymer, 51, 2435 (2010).

8. D.Q. Chen, Y.Z. Wang, X.P. Hu, D.Y. Wang, M.H. Qu and B. Yang, Polym. Degrad. Stab., 88, 349 (2005).

9. M. Jikei and M.A. Kakimoto, Prog. Polym. Sci., 26, 1233 (2001).

10. Y.H. Kim, J. Polym. Sci. A Polym. Chem., 36, 1685 (1998).

11. C. Gao and D. Yan, Prog. Polym. Sci., 29, 183 (2004).

12. C.R. Yates and W. Hayes, Eur. Polym. J., 40, 1257 (2004).

13. Y. Lin, K.Y. Zhang, Z.M. Dong, L.S. Dong and Y.S. Li, Macromolecules, 40, 6257 (2007).

14. Y. Hong, S.J. Coombs, J.J. Cooper-White, M.E. Mackay, C.J. Hawker, E. Malmström and N. Rehnberg, Polymer, 41, 7705 (2000).

15. R. Mezzenga, C.J.G. Plummer, L. Boogh and J.-A.E. Manson, Polymer, 42, 305 (2001).

16. R. Bhardwaj and A.K. Mohanty, Biomacromolecules, 8, 2476 (2007).

17. Y. Kikkawa, H. Abe, T. Iwata, Y. Inoue and Y. Doi, Biomacromolecules, 3, 350 (2002).

18. N. Ljungberg and B. Wesslen, Biomacromolecules, 6, 1789 (2005).

19. Y. Lin, K.Y. Zhang, Z.M. Dong, L.S. Dong and Y.S. Li, Macromolecules, 40, 6257 (2007).

20. R. Ratz and O.J. Sweeting, J. Org. Chem., 28, 1608 (1963).

21. L.C. Wang, J.Q. Jiang, P.K. Jiang and J.H. Yu, J. Polym. Res., 17, 891 (2010)

22. M.J. Sinclair and J.E. Watts, Fire Retardant Intumescent Coating, US Patent 7,217,753 (2003). 